\title{
Current Situation and Development Countermeasures of Marine Knowledge Education in Primary and Secondary Schools in Hainan Province
}

\author{
Lishan Huang \\ Law School of Hainan University, Haikou, Hainan Province, China, 570228 \\ 1611021604@qq.com
}

Keywords: Marine Knowledge Education, Primary and Secondary Schools, Development Countermeasures

\begin{abstract}
As the ocean occupies about 70\% of the earth's surface, and every country in the world strives for maritime rights and interests actively, which shows ocean is vital for the country. The 21st century is an era of the ocean. In the context of new media, we should firmly grasp this opportunity. From the sample survey of the students who are from primary and middle school in Hainan Province, we can understand the importance of teaching marine knowledge to the primary and middle school students. This paper analyzes the problems existing in the implementation mode of marine knowledge education and analyzes its reasons, and studies the development countermeasures of marine knowledge education. In particular, it proposes specific ways to use the new media to carry out marine knowledge education. In the context of new media, the education of marine knowledge for primary and middle students can not only stimulate the enthusiasm and interest in acquiring marine knowledge, but also lay the foundation for the formation of a multilevel and systematic marine education.
\end{abstract}

\section{Introduction}

As new media has become more and more important in the modern media industry, its application in various industries has become more and more extensive. Meanwhile, marine knowledge education is an important content of patriotism education for students, and a vital point for students to learn about territorial resources education, environmental protection education and science popularization education. Besides, it is an indispensable course content for higher education. The arrival of the ocean era has further promoted the rise of marine education. Therefore, in the context of new media, the development of marine knowledge education for primary and middle students has become a requirement of the times.

From the sample survey, we can know about the current situation of the primary and middle students' cognition of the ocean. Taking the new media as the background, we analyze the current implementation model of marine knowledge education, and propose countermeasures for the future development of marine knowledge education in primary and middle schools. On the basis of raising marine awareness of primary and middle students, we aim to strengthen the education of marine knowledge, and to lay a foundation for building a multilevel marine education system and cultivating marine talents. Through social research, we study the breadth and depth of the impact of new knowledge dissemination methods on marine education in the context of the information age. And in reality, according to the unbalanced teaching quality and resource allocation in primary and middle schools, we will study the development mode of providing new opportunities for primary and middle students to learn marine knowledge through different media platforms, which will effectively improve the marine knowledge reserve of primary and middle students, and improve research through the feasibility of the results enables the development countermeasures for marine knowledge education in primary and secondary schools to be widely promoted. 


\section{The Current Problems of Marine Knowledge Education in Primary and Secondary Schools}

\subsection{Insufficient Resources of Marine Knowledge Education in Schools}

Textbooks, the main carrier of marine knowledge education in primary and middle schools, are mainly used as non-major courses such as nature and geography. But in the modern society with high economic and technological development, teaching with paper is obviously unable to meet the needs of the students who are full of curiosity about science and technology. Nowadays, for the primary and middle students, they need three-dimensional, dynamic, development, diverse teaching of marine knowledge. Although China's expenditure on education has been increasing, and the basic teaching facilities of primary and middle schools have been constantly balanced and improved, it is still insufficient in the field of marine knowledge education. Therefore, the monotonous, static and one-sided marine knowledge education will not stimulate students' leaning interest and their motivation, which obviously is not conducive to the popularization of marine knowledge and the enhancement of marine awareness.

\subsection{The Textbooks are Less Involved in Marine Knowledge}

Since the 21st century, marine-related content has been involved in primary and middle education, but the degree of popularization is far from enough, and in many areas marine-related content are only mentioned in history or geography textbooks. The teaching materials basically do not cover the situation concerning the rights and interests of the sea, as well as deep-level contents such as the marine exclusive economic zone, maritime law and coastal defense construction. The only content is usually out-fashioned and lacks attraction to primary and middle students. In addition, there are few presentations on marine life, marine science and technology, marine culture, etc., and students are unable to fully understand the richness and charm of marine resources. Students cannot fully understand the richness and charm of marine resources, which is naturally impossible to obtain more effective teaching effect.

\subsection{Lack of Teachers in the Marine Professional Field}

Some primary and middle schools teach marine knowledge through courses such as nature, geography, and history. But, in fact, those teachers rarely have a marine professional background, and their own understanding of marine knowledge is extremely limited. And for teachers who are not self-motivated, they may simply teach textbook content purely, and they will not focus on the country's ocean strategy, maritime disputes, marine rights protection, and ocean economy. Some schools even arrange other curriculum teachers to teach subjects like nature, which may not be familiar with the contents of the textbook. This shows the lack of marine professional teachers. The so-called professionalism of the professions shows the lack of marine professional teachers.

\subsection{The Unreasonable Curriculum Setting}

As we all know, Chinese, mathematics and English are the main courses all the time. The time schedules are always given priority to these courses. Even some sports and self-study courses have to do the same thing. Of course, the emphasis on such courses is reasonable, because they have a fundamental role in the growth of student, in the aspect of literacy and personality. However, we cannot ignore education of marine knowledge, and we have always advocated comprehensive development and quality education. As the role of the oceans in economic and social development becomes more prominent, it is clear that if we continue to adhere to such a teaching model, it will be behind the development of the times. It is very urgent that the teaching course of ocean knowledge and the practice course should be required in the times.

\section{The reasons of the problems in marine knowledge education in primary and middle school}

\subsection{Lack of funds for marine knowledge education in schools}

The school has a strict management system for the use of teaching funds. If the original 
educational funds of the school are not increased, we need to make sure the expense of regular teaching activities. At the same time, when we using the new media to conduct marine knowledge teaching, which obviously will place a greater burden on schools. Under tight financial constraints, schools are bound to reduce other expenditures in order to ensure that, which will have a negative impact on the management of schools or even on other learning conditions of primary and middle school students. In addition, even if the school has sufficient funds to support the development of marine knowledge education, the school may not be willing to invest in this field. After all, the issue of school enrollment rate is more concerned by schools.

\subsection{The Weak Awareness of the Ocean Right}

As we Chinese in history always attach importance of the land, which is accompanied by an indifference to the marine culture, especially the policy of shutting down the country after Ming Dynasty, which keep us away from sea in thought and action. At the same time, people's daily life involves few opportunities with ocean, and our food, clothing, shelter, and transportation can still be guaranteed. Therefore, although the country strongly calls for the strategy of "ocean power”, most of the people still fail to give more attention. No matter you are a parent or a teacher, everyone needs to aware of maritime power. If we fail, then the cultivation of marine consciousness and the education of marine knowledge of primary and middle students will be even more difficult.

\subsection{Lack of Marine Professionals to Participate in Primary and Middle school Education}

In general, the marine knowledge education in our basic education system has an obvious fault with the marine professional education in higher education, some of which are not related. It is easy for most marine professionals to assume that what they have learned is unnecessary or underutilized in primary and secondary education, therefore, they are more willing to engage in higher education or to work in enterprises and other places. The Ministry of Education does not have the policy support to guide the flow of talents for this area. The school has no such awareness of recruiting talents. As a result, marine professionals are hardly involved in primary and middle education.

\subsection{The Serious Tendency of Exam-Oriented Education}

The problem of exam-oriented education has been criticized but always exists. Parents, schools, and even students themselves have to put their test scores in a high position. Performance is of course one of the important criteria to assess the comprehensive quality of students, but sometimes it is clearly contrary to the educational concept to be required and evaluated as the sole criterion for students. Of course this is also inseparable from the assessment content of the school by the Education Bureau and the focus on the community on the education of the school. Therefore, the examination-oriented problem itself is a problem co-produced by multiple subjects. Under this educational concept and method, the Marine knowledge education obviously cannot get due attention and sufficient investment.

\section{The Countermeasures under the Background of New Media}

\subsection{Improve the Level of Marine Knowledge Education in Primary and Middle Schools}

If the government is to increase the funding for marine education in primary and middle schools, it can set up a marine education research fund to encourage teachers to actively study and develop more efficient marine education models, and to provide schools with good marine education facilities and materials, so that primary and secondary students can early access to marine science and technology, and higher education in the training of marine personnel. The marine knowledge in the existing subject textbooks should be greatly increased, or a new course of marine knowledge education should be added, and the teaching time should be allocated reasonably to ensure that students have enough time to learn more about marine knowledge. At the same time, it is necessary to encourage marine professionals to join the primary and middle school education, and provide them with a good talent introduction condition and development platform to enable them to fully utilize their talents. 


\subsection{Raising the National Awareness of the Ocean}

We will give full play to the role of public resources in marine education, carry out scientific planning for existing marine museums, science and technology museums, strengthen the construction of marine specialized museums, marine science education network, etc. We will increase lectures on marine science and technology, provide marine education with more abundant educational resources and means, and strive to increase the popularity of marine education for all. At the same time, the news media should spread the protection of maritime rights, the marine environment, and the dynamics of marine science and technology. Columns should be set up in popular science magazine and education program to comprehensively explain the Marine knowledge, providing a good learning platform for the national Marine education.

\subsection{Teaching Marine Knowledge Education with New Media}

At first, we will make some interesting design to allow primary and middle students to learn about the ocean in a relaxed environment. There is a unique geographical advantage in Hainan Province, and by uploading articles, videos and photos about the ocean, which stimulates student's enthusiasm to learn about marine knowledge and achieves the initial goal of "pro-sea". Besides, regularly organizing students to watch excellent ocean documentaries, can not only attract students into the ocean world gradually, but also let us learn about different students' interests and hobbies on different marine knowledge topics, so as to facilitate future investigation, research, analysis and summary. What's more, we can develop some encouraging projects and adapt to the psychological acceptance of primary and middle students, such as a daily question. Students not only can reduces the learning burden, but also accumulates marine knowledge. In this platform, student's performance can be shown by his or her participating in the project, and rewards are given according to their participation and length of study.

\section{Conclusion}

The arrival of the ocean era and the rapid development of new media have made marine education an inevitable topic, making the use of new media a natural means. Rome wasn't built in a day, so it takes great effort to build the project which based on the marine knowledge of primary and middle students education. When we utilize the characteristics of new media to enhance the diversity and interest of the content and form of marine knowledge education, when we cultivate the marine awareness of young children and the ability to learn marine knowledge from primary education, special attention should be paid to marine knowledge education in primary and middle schools. When the expertise of marine science and the law of the sea is integrated, it is no longer difficult to cultivate national marine awareness. In the fields of education, the goal of "Pro-Ocean, Know the Ocean, Love the Ocean” is achieved, so that students can realize the marine values, thus providing a sustainable source and motivation for the development of marine talents.

\section{Acknowledgement}

2017 Hainan University Undergraduate Innovation and Entrepreneurship Training Program, "Development Strategies for Marine Knowledge Education in Primary and Secondary Schools under the Background of New Media”, Item No. 201710589143.

\section{References}

[1] Mao Qihuang. Development of Marine Education and Strategy of Ocean Powerful Country [J]. Advance Forum, 2012(3).

[2] Yu Hai. On the measures to safeguard China's maritime rights and interests under the current situation [J]. China Water Transport (second half), 2010 (4).

[3]Zhang Mengyan. On the Cultivation of Marine Awareness in the Perspective of Basic Education 
[J].Asia-Pacific Education, 2016, (05).

[4] Wu Weiwei, Wang Yongjun. On the era value of developing marine education [J]. Journal of Changchun Normal University, 2014, 33(03).

[5] Hu Yiyun, Shao Feng, Jin Yunliang. The Ways of Cultivating National Ocean Consciousness under New Media Technology [J]. Special Zone Economy, 2018(08). 